\title{
MULTIPLICATION RULES FOR POLYNOMIALS
}

\author{
MELVYN B. NATHANSON
}

\begin{abstract}
It is proved that the polynomial solutions of the functional equation

$$
F(z) F(z+1 / \sqrt{a})=F\left(\sqrt{a} z^{2}+(b / \sqrt{a}+1) z+c / \sqrt{a}\right)
$$

are precisely $\left(a z^{2}+b z+c\right)^{n}$ if $b^{2}-4 a c \neq 0$ and $(\sqrt{a} z+b / 2 \sqrt{a})^{n}$ if $b^{2}-4 a c=0$.
\end{abstract}

Let $F(z)=a z^{2}+b z+c$, where $a, b, c \in \mathbf{C}$ and $a \neq 0$. Then $F(z)$ satisfies the "multiplication rule"

$$
F(z) F(z+1 / \sqrt{a})=F\left(\sqrt{a} z^{2}+(b / \sqrt{a}+1) z+c / \sqrt{a}\right) .
$$

In particular, if $a=1$, then $F(z) F(z+1)=F\left(z^{2}+(b+1) z+c\right)$. From this observation, Cohen, Erdös, and Nathanson [2] deduced that if $F(z)=z^{2}$ $+b z+c$ with $b, c \in \mathbf{Z}$, and if $F(n) \geqslant 1$ for all $n \geqslant 1$, then infinitely many terms of the sequence $\{F(n)\}_{n=1}^{\infty}$ are "composite" in the sense that they factor into the product of strictly smaller terms of the sequence.

If $F(z)=z^{2}+1$, then the multiplication rule (1) implies that $F(z) F(z+$ 1) $=F\left(z^{2}+z+1\right)$. H. N. Shapiro [3] proved the converse: If $F(z)$ is a nonzero polynomial such that $F(z) F(z+1)=F\left(z^{2}+z+1\right)$, then $F(z)=$ $\left(z^{2}+1\right)^{n}$. In this note I extend Shapiro's result to the general multiplication rule (1): If $b^{2}-4 a c \neq 0$, then the only nonzero polynomial solutions of (1) are $\left(a z^{2}+b z+c\right)^{n}$; if $b^{2}-4 a c=0$, then the only nonzero polynomial solutions of (1) are $(\sqrt{a} z+b / 2 \sqrt{a})^{n}$. This result depends on the following Lemma, first proved by the author for real $d$, and then extended by B. J. Birch [1] to complex $d$ with a remarkably simple proof.

LEMMA. Let $d \in \mathrm{C}$, and let $S_{d}$ be a finite, nonempty set of complex numbers such that $z \in S_{d}$ implies $z^{2} \pm z-d \in S_{d}$. Then $\{ \pm \sqrt{d}\} \subseteq S_{d}$. Moreover, $S_{0}=\{0\}$.

Proof. The set $S_{d}$ is finite and nonempty, and so there exists $z \in S_{d}$ with $|z|$ maximal. Since $z^{2} \pm z-d \in S_{d}$, it follows that $|z| \geqslant \max \left\{\left|z^{2}+z-d\right|\right.$, $\left.\left|z^{2}-z-d\right|\right\}$. But $\left(z^{2}+z-d\right)-\left(z^{2}-z-d\right)=2 z$, hence $|z|=\mid z^{2}+z$ $-d|=| z^{2}-z-d \mid$. This is possible only if $z^{2}-d=0$, hence $z=\sqrt{d}$ or $z=-\sqrt{d}$. But if $\sqrt{d}$ (resp. $-\sqrt{d}$ ) belongs to $S_{d}$, then so does $-\sqrt{d}$ (resp.

Received by the editors August 3, 1977.

AMS (MOS) subject classifications (1970). Primary 10B20, 12D05, 39A15.

Key words and phrases. Functional equations, multiplication rules, polynomial factorization. 
$\sqrt{d}$ ). Moreover, if $z \in S_{0}$, then $|z| \leqslant 0$, and so $S_{0}=\{0\}$.

TheOREM 1. Let $a, b, c \in \mathbf{C}, a \neq 0$, and let $F(z)$ be a nonzero polynomial such that

$$
F(z) F(z+1 / \sqrt{a})=F\left(\sqrt{a} z^{2}+(b / \sqrt{a}+1) z+c / \sqrt{a}\right) .
$$

Then for some $n \geqslant 0$,

$$
F(z)= \begin{cases}\left(a z^{2}+b z+c\right)^{n} & \text { if } b^{2}-4 a c \neq 0 \\ (\sqrt{a} z+b / 2 \sqrt{a})^{n} & \text { if } b^{2}-4 a c=0\end{cases}
$$

Proof. If $F(z)=k$ is a nonzero constant satisfying (1), then $k^{2}=k$ and so $F(z)=1$. If $F(z)$ is a nonconstant polynomial satisfying (1), then $G(z)=$ $F(z / \sqrt{a}-b / 2 a)$ satisfies

$$
G(z) G(z+1)=G\left(z^{2}+z-d\right),
$$

where $d=\left(b^{2}-4 a c\right) / 4 a$. The set $S_{d}$ of roots of the polynomial $G(z)$ is a finite nonempty set of complex numbers, and (2) implies that $S_{d}$ is closed under $z \mapsto z^{2} \pm z-d$. By the Lemma, $S_{d}$ contains $\pm \sqrt{d}$. If $d \neq 0$, then $G(z)$ can be written in the form $G(z)=\left(z^{2}-d\right)^{n} H(z)$, where $n \geqslant 1$ and not both $\pm \sqrt{d}$ are roots of $H(z)$. Since both $G(z)$ and $\left(z^{2}-d\right)^{n}$ satisfy the functional equation (2), it follows that $H(z)$ also satisfies (2). But if $H(z)$ is nonconstant, then the set of roots of $H(z)$ must also contain both $\pm \sqrt{d}$ which is impossible. Therefore, $H(z)$ is constant, hence $H(z)=1$, and $G(z)=\left(z^{2}-d\right)^{n}$. Then

$$
F(z)=G(\sqrt{a} z+b / 2 \sqrt{a})=\left(a z^{2}+b z+c\right)^{n} .
$$

If $d=0$, then $S_{0}=\{0\}$ and $G(z)=z^{n}$, hence

$$
F(z)=G(\sqrt{a} z+b / 2 \sqrt{a})=(\sqrt{a} z+b / 2 \sqrt{a})^{n} .
$$

This proves the theorem.

If $F(z)=a z^{2}+b z+c$ with $a \neq 0$, then the multiplication rule (1) implies that

$$
F(z) F(z+1 / \sqrt{a})=F(z+F(z) / \sqrt{a}) .
$$

This observation has the following converse.

THEOREM 2. Let $a \neq 0$ and let $F(z)$ be a nonconstant polynomial such that (3) holds. Then $F(z)=a z^{2}+b z+c$.

Proof. Let $F(z)$ satisfy (3). If $F(z)$ has degree $n$, then the left side of (3) has degree $2 n$ and the right side of (3) has degree $n^{2}$. Therefore, $n=2$. If $F(z)$ has leading coefficient $A$, then the left side of (3) has leading coefficient $A^{2}$ and the right side of (3) has leading coefficient $A(A / \sqrt{a})^{2}=A^{3} / a$. Therefore, $A=a$, and so $F(z)=a z^{2}+b z+c$ for some $b, c \in \mathbf{C}$. This proves the Theorem.

Problems. The function $F(z)$ satisfies a multiplication rule if there exist 
polynomials $\alpha(z), \beta_{1}(z), \ldots, \beta_{t}(z)$ with $t \geqslant 2$ and degree $\alpha \geqslant 2$ such that $F(\alpha(z))=F\left(\beta_{1}(z)\right) \cdots F\left(\beta_{t}(z)\right)$. Which polynomials $F(z)$ satisfy a multiplication rule? In particular, does $z^{n}+1$ satisfy a multiplication rule for any $n \geqslant 3$ ? Similar questions can be asked about more general multiplication rules of the form

$$
F\left(\alpha_{1}(z)\right) F\left(\alpha_{2}(z)\right) \cdots F\left(\alpha_{s}(z)\right)=F\left(\beta_{1}(z)\right) \cdots F\left(\beta_{t}(z)\right)
$$

where $\alpha_{i}(z) \neq \beta_{j}(z)$ for $1 \leqslant i \leqslant s$ and $1 \leqslant j \leqslant t$, and degree $\alpha_{1}+\cdots+$ degree $\alpha_{s} \geqslant 2$.

The only polynomial solutions of the functional equation $F(z) F(z+1)=$ $F(z+F(z))$ are the monic quadratic polynomials. What are the nonpolynomial solutions of this equation?

\section{REFERENCES}

1. B. J. Birch, personal communication.

2. S. D. Cohen, P. Erdös and M. B. Nathanson, Prime polynomial sequences, J. London Math. Soc. 14 (1976), 559-562.

3. H. N. Shapiro, The powers of $x^{2}+1$, Math. Mag. (to appear).

Department of Mathematics, SOUTHERn Illinois University, Carbondale, ILlinois 62901

Current address: Department of Mathematics, Harvard University, Cambridge, Massachusetts 02138 